TRABAJOS DE PREHISTORIA

53, n. ${ }^{\circ} 1,1996$, pp. $155-162$

\section{UNA CUENTA SINGULAR VITRIFICADA DE TIPO ORIENTALIZANTE ENTRE LOS DEPÓSITOS FUNERARIOS DE UN TÚMULO CATALÁN DEL BRONCE MEDIO: EL MONUMENTO I DE EL BOSC DE CORREÀ (L'ESPUNYOLA, BERGUEDÀ, CATALUÑA)}

\author{
AN OUTSTANDING GLASS BEAD OF \\ ORIENTAL TYPE AMONGST THE \\ FUNERARY STOCKS IN A CATALAN \\ BARROW OF THE MIDDLE BRONZE \\ AGE: MONUMENT I OF THE EL BOSC \\ OF CORREÄ (L'ESPUNYOLA, \\ BERGUEDÄ, CATALONIA)
}

\section{JORDI ROVIRA I PORT (*)}

\section{RESUMEN}

En este trabajo se analiza una interesante cuenta de pasta vítrea del Bronce Medio localizada en un túmulo catalán y publicada en 1927 por J. Serra Vilaró del Museo de Solsona. La pieza muestra una decoración oculada y se trata, sin duda, de un elemento importado desde el Mediterráneo oriental o central, quizás a través del comercio micénico o circunmicénico.

\footnotetext{
ABSTRACT

In this paper we examine an interesting glass bead of the Middle Bronze Age found in catalan barrow and published in 1927 by J. Serra Vilaró, director of the Solsona

(*) Museu d'Arqueologia de Catalunya. Passeig de Santa Madrona, 39-41. 08038 Barcelona.

El artículo fue remitido en su versión final el 22-I-96.
}

museum. This object has an eyepieces decoration and, undoubtedly, it is an imported element coming from the eastern or central Mediterranean Sea, perhaps by means of Mycenaean or circummycenaean trade.

Palabras clave: Cuenta. Pasta vítrea. Ámbar. Bronce Medio. Túmulo. Oculada. Comercio micénico. Circunmicénico.

Key words: Glass bead. Amber. Middle Bronze Age. Barrow. Eyepieces. Mycenaean/circummycenaean trade.

\section{INTRODUCCIÓN}

En el complejo panorama de los materiales suntuarios y de prestigio que suelen acompañar a los objetos más comunes que constituyen los depósitos sepulcrales de la Edad del Bronce del 
occidente europeo, subyace una importante información indispensable para llegar a conocer el entramado de relaciones comerciales y contactos entre los diversos territorios europeos y una parte substancial de las áreas mediterráneas. No hace falta incidir aquí en la ya clásica polémica sobre el origen de las piezas manufacturadas en ámbar de fechación pre y protohistórica, y tampoco es nuestra intención abordar aquí en profundidad la problemática de los objetos elaborados en distintos tipos de pasta vítrea que aparecen cada vez con mayor asiduidad y cuantía en un gran número de yacimientos de la Europa occidental. De hecho, todas estas cuestiones han sido ya analizadas por nosotros mismos en un denso trabajo que ha precedido a otros futuros que elaboraremos (Rovira, 1995).

Así, el objeto del artículo que ahora nos ocupa es presentar, reestudiar y valorar a la luz

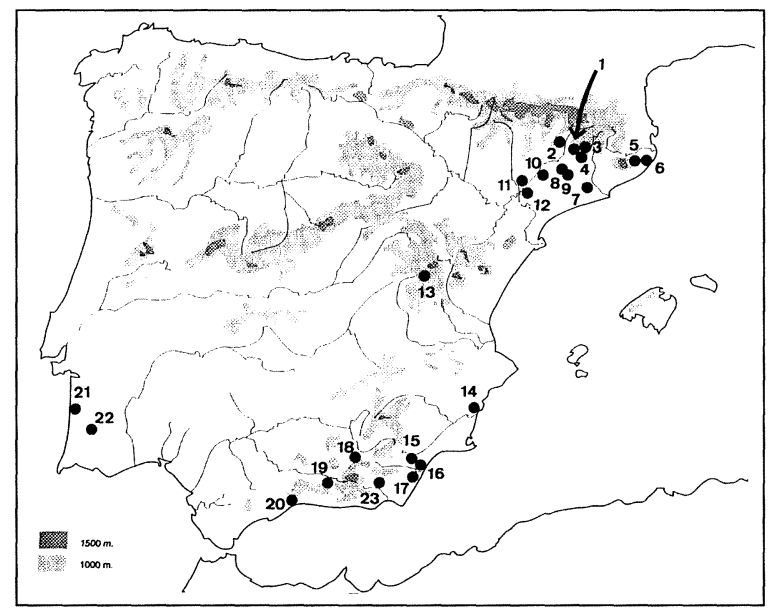

Fig. 1. Localización de los yacimientos que han proporcionado elementos de pasta vítrea en la Península Ibérica datables entre el Calcolítico/Bronce Antiguo y el siglo VIII a.C. La flecha señala la ubicación del túmulo I de El Bosc de Correà.

La relación de los yacimientos representados en el mapa es la siguiente:

1. El Bosc (L'Espunyola, Barcelona); 2. Cal Colau (Montanissell, Lérida); 3. Can Maurí (Berga, Barcelona); 4. Clot dels Morts I (Montmajor, Barcelona); 5. Cova d'En Daina (Romanyà de la Selva, Gerona); 6. Puig-ses-Forques (Sant Antoni de Calonge, Gerona); 7. Roca del Frare (La Llacuna, Barcelona); 8. L'Auritori (Guissona, Lérida); 9. Bell-Pla (Guissona, Lérida); 10. La Pedrera (Térmens, Lérida); 11. Tossal de Carretelà (Aitona, Lérida); 12. Tossal de Solibernat (Torres de Segre, Lérida); 13. Pajaroncillo (Cuenca); 14. Peña Negra (Alicante); 15. Fuente Álamo (Almería); 16. Herrerías (Almería); 17. El Argar (Almería); 18. La Sabina (Granada); 19. Cerro de la Encina (Granada); 20. Vélez Málaga (Málaga); 21. Sines (Portugal); 22. Atalaia (Ourique, Portugal); 23. El Peñón de la Reina (Alboloduy, Almería). de nuestros conocimientos actuales, un elemento ornamental y simbólico elaborado en pasta vítrea y procedente de los ajuares sepulcrales de un túmulo catalán (Fig. 1), excavado, estudiado y publicado hace casi setenta años - en 1927- por Mossén J. Serra Vilaró (1927), a la sazón director del Museu Arqueològic Diocesà de Solsona.

\section{EL ELEMENTO ESTUDIADO Y SU ANÁLISIS}

La pieza en cuestión consiste en una cuenta subesferoidal fuertemente achatada en sus caras superior e inferior (1). La cuenta aparece atravesada axialmente por una bien visible perforación cilíndrica que se ensancha levemente en ambos extremos de salida al exterior y fue elaborada a partir de una pasta muy maleable, posteriormente vitrificada - ahora de color marrón rojizo/verdoso-, a semejanza de las piezas de vidrio rudimentario o pasta vítrea. Muestra en su desarrollo lateral una singular y compleja ornamentación consistente en surcos y ondulaciones marcadas profundamente en la masa moldeada, en un proceso previo a la vitrificación. Se trata de ondulaciones longitudinales que constituyen una cenefa compuesta de tres triángulos, pseudohojas o motivos similares relacionados con la ondulación que conforma el motivo superior decorativo de la pieza. Hay que resaltar que esta particular combinatoria está al servicio de un efecto global en la cuenta que persigue realzar el motivo principal dibujado en las caras achatadas, es decir, delinear perfectamente, tanto en la cara superior como en la inferior, la silueta de un oudja, un oculus, cuya pupila estaría constituida por la perforación circular central (Rovira, 1982). Por lo que respecta al iris, éste se insinúa mediante las curvaturas rebajadas, y, en lo que concierne al dibujo del perímetro ocular -fuertemente rasgado o almendrado en una

(1) A pesar de los años transcurridos, no queremos perder la ocasión de dejar aquí constancia de nuestra gratitud para con el ya extinto Dr. Antoni Llorens, antiguo director del Museu Arqueològic Diocesà de Solsona por su colaboración en pro de facilitar el estudio de la pieza que nos ocupa.

T. P., 53, n. ${ }^{\circ} 1,1996$ 

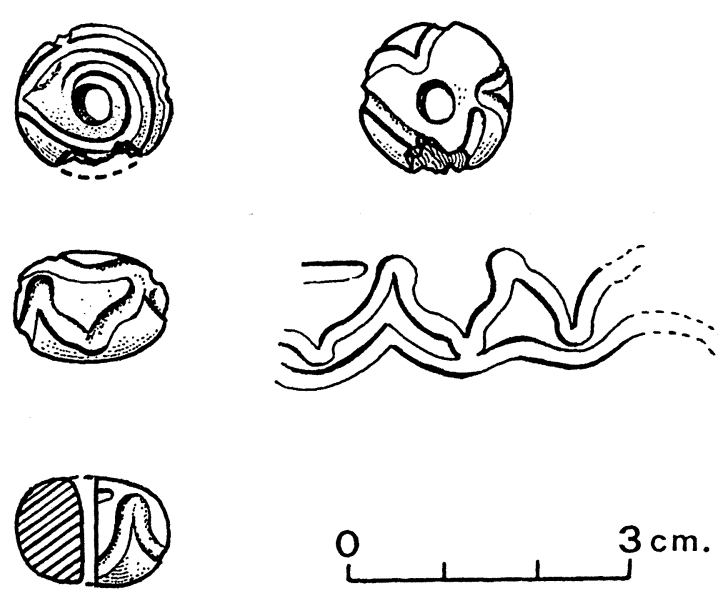

Fig. 2. Anverso, reverso y desarrollo lateral de la cuenta de pasta vitrificada con decoración oculada del túmulo I de El Bosc de Correà (Berguedà, Cataluña).

de las caras y menos marcado en la otra-, aparece representado con total claridad y expresionismo (2) (Fig. 2).

En cuanto a las características físico-químicas de este elemento oculado, su análisis mediante espectrografía de rayos infrarrojos (3), ofreció como resultado una composición que engloba carbonato cálcico, cuarzo/sílice y paratacamita (cloruro básico de cobre en forma pulverulenta sin cristalización) junto con algún resto orgánico carbonoso. Así, la paratacamita cumple aquí un papel similar al que poseen los óxidos de cobre en piezas similares, mientras que el carbonato cálcico delata una probable procedencia marina, concretamente de una zona calcárea con inclusión de micronummulites. Por otro lado, la materia que compone la cuenta presenta un desprendimiento gaseoso a una temperatura superior a los $850 / 900^{\circ} \mathrm{C}$, y su aspecto al microscopio confirma la existencia

(2) La cuenta oculada de El Bosc fue clasificada como una cuenta de ámbar más en la relación de materiales efectuada en su momento por J. Serra Vilaró, y así consta en su monografía del año 1927 en la que se habla de 20 cuentas de resina cuando en realidad se exhumaron 19. Ya en 1972 y con motivo de una nueva reordenación y rotulación de materiales arqueológicos del Museo de Solsona, advertimos las características singulares de este elemento y el hecho de que había sido elaborado en pasta vítrea, y así lo hicimos constar en 1974 (Rovira Port, 1982).

(3) El análisis espectrográfico fue efectuado por el analista Sr. P. de la Morena, a quien agradecemos su colaboración. de abundantes burbujas bien definidas, así como una vitrificación superficial notable. De hecho, estamos en presencia de un elemento de pasta vítrea o vitrificada, de un vidrio rudimentario. Su peso específico es $\mathrm{Pe}<2.65$ y su peso total antes de la toma de la muestra analizada llegaba hasta los 3,0150 gramos. Sus dimensiones son las siguientes: $16 \mathrm{~mm}$ de diámetro máximo por $12 \mathrm{~mm}$ de altura. Ya en el momento de su exhumación, la cuenta apareció ligeramente fragmentada e incompleta (Fig. 3).

\section{EL DEPÓSITO FUNERARIO. LOS MATERIALES ACOMPAÑNANTES}

La cuenta con decoración oculada procede del túmulo número I de la zona de El Bosc de Correà, municipio de L'Espunyola, comarca del Berguedà, provincia de Barcelona. De este modo, aunque el sepulcro, ya antes de su excavación en 1927, había sufrido abundantes remociones y rebuscas incontroladas, aún fue posible recuperar un muestrario significativo de los ajuares sepulcrales que acompañaban a las quince inhumaciones existentes en el monumento.

Así, la pieza que nos ocupa fue exhumada juntamente con fragmentos de vasos con decoración incisa y pseudo impresa, asimilables al complejo campaniforme, otras producciones cerámicas con decoraciones unguiculares y digitaciones, vasos carenados con apéndices de botón cilíndricos, y restos de talla junto con un fragmento de hoja de sílex. Por lo que respecta a los objetos metálicos, aparecieron un brazalete macizo cuasi circular, abierto, otro brazalete espiraliforme, pequeños colgantes espiraliformes, anillos y apliques, junto con punzones biapuntados y otros vestigios, caso de un posible remache o vástago de sujeción. En cuanto a otros elementos ornamentales y de prestigio, señalemos la exhumación de un centenar de dentalia, trece ejemplares de columbellae y tres cuentas discoidales de cardium. A éstos habría que añadir el interesante hallazgo de diecinueve cuentas de distintos tamaños y morfologías confeccionadas en ámbar. Así, se localizaron piezas de collar y colgante de formas ovaladas, planas, tipo ba- 


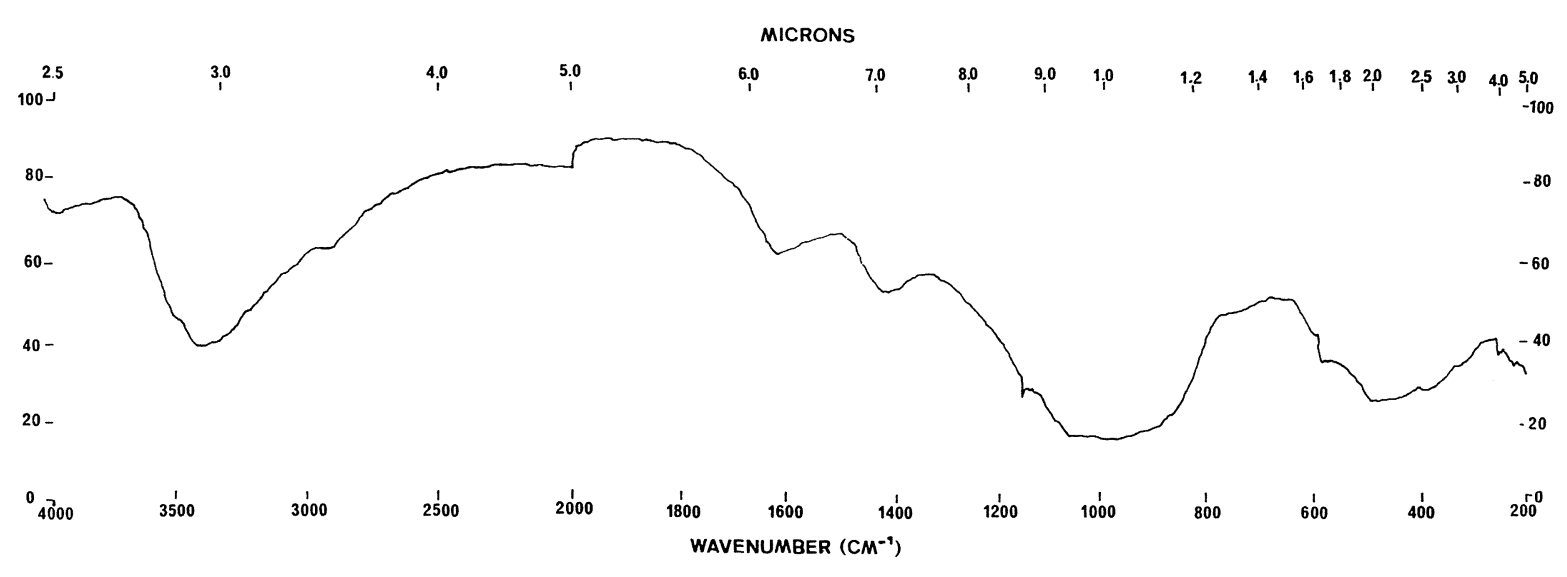

Fig. 3. Espectrograma resultante del análisis de la cuenta de El Bosc de Correà (Berguedà, Cataluña), según P. de la Morena.

rril, cuentas alargadas y de secciones facetadas, entre triangulares, cuadrangulares y pentagonales. Sus longitudes originarias oscilaban entre los 10/11 mm, algunas de $12,19 \mathrm{~mm}$ y hasta unas longitudes máximas aproximadas de $24 / 25 \mathrm{~mm}$. En su totalidad, las cuentas se hallaban vaciadas longitudinalmente mediante una pequeña perforación cilíndrica o bitroncocónica. El túmulo I de El Bosc proporcionó así el conjunto más numeroso e interesante de piezas de ámbar exhumado hasta la fecha en Cataluña (Lám. I).

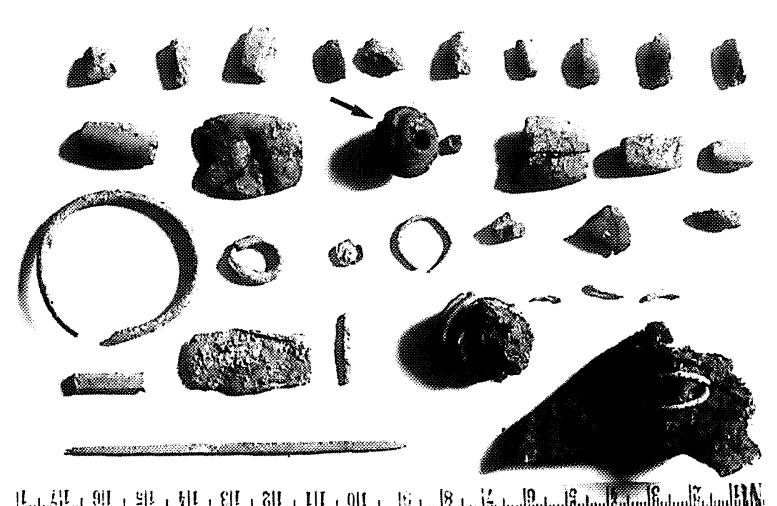

Lám. I. Fotografía/inventario de algunos de los materiales exhumados en los depósitos funerarios del túmulo I de El Bosc de Correà. En las hiladas superiores se aprecian las distintas cuentas de ámbar aparecidas. La flecha señala la cuenta de pasta vítrea oculada. Según J. Serra Vilaró. Cortesía de A. Llorens + y Museu de Solsona.

\section{EL MONUMENTO SEPULCRAL Y LA ARQUITECTURA FUNERARIA DEL BRONCE ANTIGUO/ MEDIO DE CATALUÑA}

A lo largo de un buen número de décadas, desde comienzos de siglo y, sobre todo, desde algunas de las primeras sistematizaciones sobre los sepulcros megalíticos catalanes y pirenaicos (Cazurro, 1912; Bosch Gimpera y Pericot, 19151920; Bosch Gimpera, 1915-1920, 1919; Gudiol y Colominas, 1923; Pericot, 1925, 1950; Serra Vilaró, 1927) hemos asistido a una constante recopilación y a un más afinado análisis tipológico y cronológico de las sepulturas confeccionadas con ortostatos de variados tamaños. Sin duda, la polémica subsiste y las sistematizaciones no siempre responden a filiaciones y agrupaciones de monumentos claramente diferenciadas $o$, incluso, consensuadas entre distintos investigadores. Por lo que respecta al monumento que nos ocupa, J. Serra Vilaró lo incluyó en su grupo "megalits amb túmul circular amb l'entrada a la vora del túmul" y aunque posteriormente, tanto Pericot (1950) como otros sistematizadores lo incluyeron sin más dentro de la denominación genérica de sepulcros megalíticos, lo cierto es que estamos en presencia de un auténtico túmulo de la plena Edad del Bronce (Rovira y Cura, 1989). Se trataría, probablemente, de un túmulo que encerraría un arca o cista con vestíbulopozo, es decir, una cámara rectangular o cuadrada, cerrada y precedida de un vestíbulo rectan-

T. P., 53, n. ${ }^{\circ} 1,1996$ 

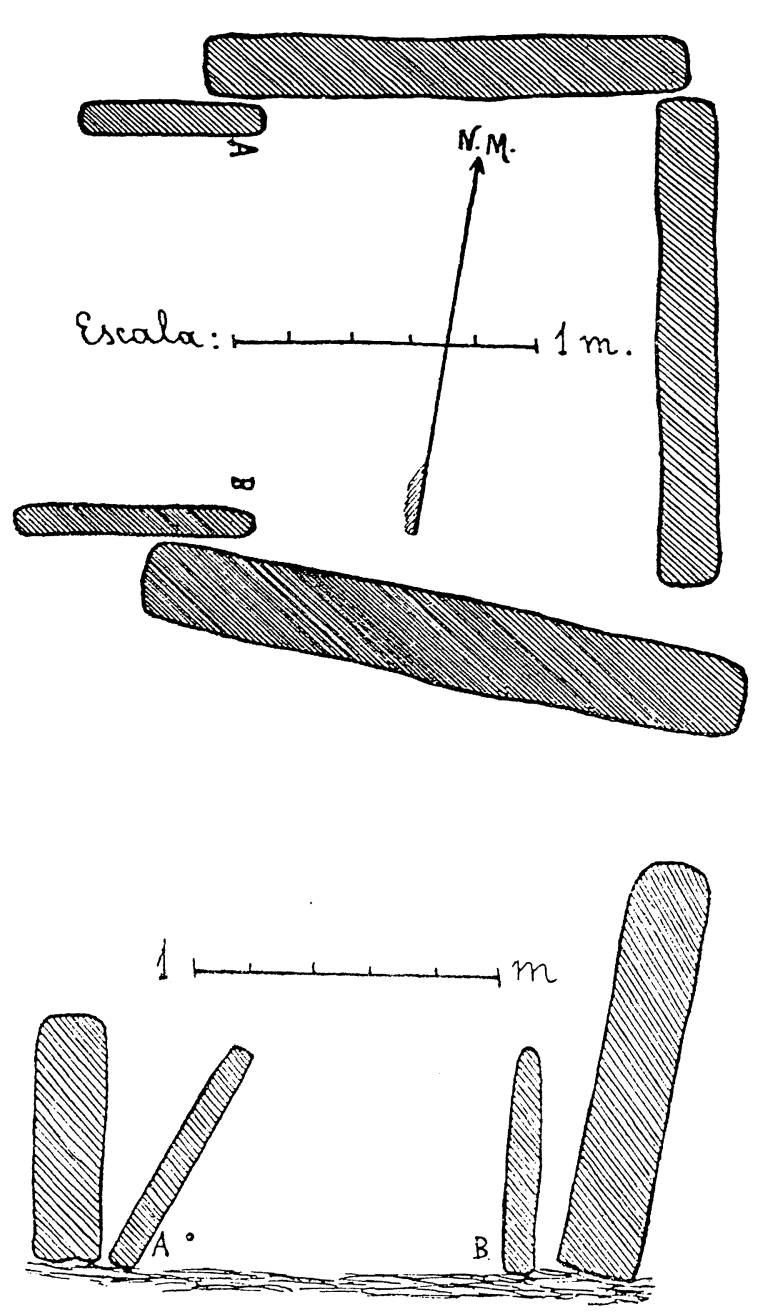

Fig. 4. Planta y sección transversal de la estructura lítica interna del túmulo I de El Bosc de Correà (Berguedà, Cataluña), según J. Serra Vilaró (1927).

gular que, funcionando a modo de pozo de acceso, posibilitaría la reutilización de la cámara sin necesidad de desmontar el túmulo que la protegía a través de un acceso - disimulado o noen la superficie de la estructura tumular. Así lo vemos en la planta y sección publicadas por $\mathrm{J}$. Serra Vilaró puesto que apreciamos en el lado oeste de la cámara central, la existencia de dos losas originariamente dispuestas en un pequeño tramo, de manera paralela a los dos grandes ortostatos norte y sur, respectivamente, de la cámara sepulcral. Se trataría de las losas A y B, componentes líticos de la mencionada antecámara de acceso (Fig. 4). De hecho, otras estruc- turas líticas internas similares a las del túmulo I de El Bosc aparecen documentadas en Cataluña a lo largo de distintos territorios, especialmente en las comarcas interiores y de poniente del Principado, y así lo constatamos en el túmulo I de la Serra de Clarena en Castellfollit del Boix (L'Anoia, Barcelona) (Castells et alii, 1983).

En efecto, precisamente este último sepulcro ejemplificaría parcialmente tanto la arquitectura como la práctica funeraria utilizadas en muchos de los túmulos catalanes de la Edad del Bronce, puesto que su cámara rectangular y su vestíbulopozo de pared seca con su estructura adyacente, nos muestran una vez más el sistema más profusamente empleado para depositar las inhumaciones en el interior de la estructura tumular. Es decir, tras la construcción del túmulo y la cubrición de la cámara central, el interior sólo era practicable a través de un pozo convertido en el único acceso delantero disponible para efectuar la introducción tanto de los restos humanos como de sus correspondientes ofrendas funerarias o lo que restase de ellos. En este sentido, el mundo tumular catalán de la plena Edad del Bronce evidencia la proliferación de las deposiciones funerarias secundarias tras un proceso previo de descarnación o incluso de cremación, al cual no eran ajenos determinados ajuares $u$ ofrendas. Así pues, los túmulos con cámara central como el que nos ocupa, muy a menudo eran construidos para ser utilizados una o pocas veces antes de sufrir una clausura definitiva del acceso al interior de la estructura funeraria.

Otros muchos túmulos - caso, por ejemplo, de los sepulcros de la Mare de Déu de Correà, Clarà, Senyús, Pla de Beret, etc.- respondían a las mismas características ahora mencionadas o a especificaciones similares a lo largo de gran parte del territorio del noreste peninsular y durante un período de tiempo que abarcó lato sensu toda la Edad del Bronce (Rovira y Cura, 1989). En definitiva, hay que adscribir al complejo tumular del segundo milenio de antes de la Era, a una gran parte de aquellas estructuras que hasta hace poco habían sido asimiladas a los grupos megalíticos compuestos de cámaras pirenaicas, cistas y cámaras con vestíbulo-pozo, y su origen hay que filiarlo en los monumentos tumulares del Neolítico Antiguo/Medio postcardial de Cataluña (Cura et alii, 1991). 

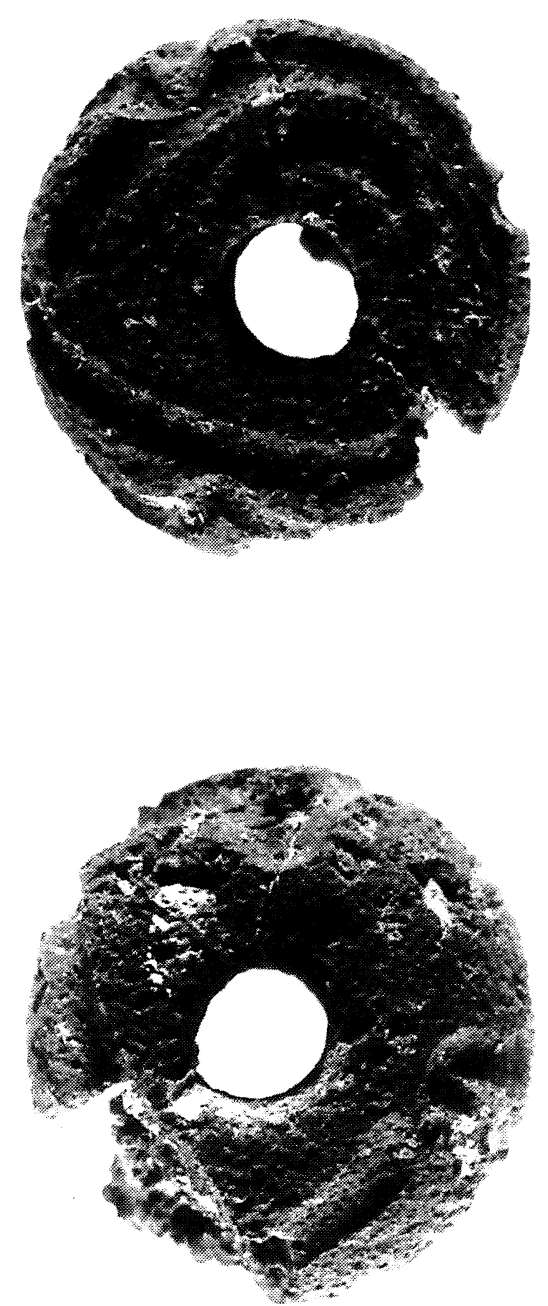

Lám. II. Fotomontaje del anverso y reverso de la cuenta oculada de El Bosc de Correà. La escala gráfica da idea de la ampliación.

\section{LA CUENTA CON DECORACIÓN OCULAR. APRECIACIONES}

La excepcionalidad de la cuenta del túmulo I de El Bosc reside esencialmente en la singularidad de su decoración global y en el simbolismo de su motivo principal. Como hemos visto, sus componentes principales - carbonato cálcico, sílice y paratacamita- coinciden grosso modo con los componentes mayoritarios existentes en muchas de las cuentas de pasta vítrea analizadas a lo largo del continente europeo, y que consisten, fundamentalmente, en sílice y cobre, en ocasiones junto con estaño, plomo, aluminio, plata, antimonio y óxido de magnesio. Ya anteriormente (Rovira Port, 1995) hemos analizado la problemática de las cuentas de pasta vítrea y sus implicaciones crono culturales por lo cual no insistiremos aquí en estas cuestiones. En cuanto a la decoración compleja que porta la pieza, podemos apreciar que ésta se obtuvo mediante la utilización de un objeto de punta roma o redondeada que se empleó presionando ligeramente la masa cuando aún se encontraba tierna y maleable, dando lugar a los motivos visibles en forma de surcos o depresiones continuadas de sección de media caña a lo largo y ancho de toda la pieza. En el proceso de decoración hubo que sujetar la pieza por su eje - que ahora se halla perforado-y, en su momento, la perforación resultante fue ensanchada y uniformada mediante pulimientación (Lám. II).

Por lo que respecta a los motivos presentes en la cuenta, ya hemos comentado que el desarrollo lateral muestra una decoración constituida por ondulaciones y sinuosidades, que conforman una cenefa compuesta básicamente por una ondulación horizontal combinada con tres pseudotriángulos o pseudohojas, y cuya misión fundamental es dar lugar a la representación ocular y arroparla.

De este modo, a pesar de las diferencias de soporte y formales y de las distintas soluciones técnicas empleadas para conseguir una imagen esencial similar, nuestra pieza de El Bosc posee un paralelo indiscutible por su iconografía principal y su simbolismo, en la cuenta discoidal de ámbar de la Grotte au Collier (Lastours, Aude) que muestra otro elemento ocular grabado como tema iconográfico omnipresente (Charles y Guilaine, 1963a y b).

\section{UNAS REFLEXIONES FINALES}

No abundaremos aquí en consideraciones sobre la iconografía ocular (AA.VV., 1994) presente en la pieza de El Bosc y su paralelo en ámbar de la Grotte au Collier, ni en apreciaciones crono culturales ya tratadas en su momento por R.P. Charles y J. Guilaine, aunque sí haremos hincapié en el paralelismo de ambos hallazgos y en las características concomitantes de 
los complejos materiales acompañantes, sobre todo, la presencia abundante de cuentas y separadores de ámbar, el origen de cuya materia prima hay que situar en el ámbito báltico, lato sensu. Otra cuestión muy distinta concierne a la manipulación del ámbar en bruto y a su transformación en elementos de prestigio sumamente codiciados por las élites locales y las jefaturas emergentes o consolidadas en toda Europa a lo largo del segundo milenio antes de nuestra Era. En este sentido, la cuenta ocular del túmulo I de El Bosc, se inscribe en una compleja y secular corriente de importaciones de elementos suntuarios que no sólo valoraba la materia prima sino que, por encima de todo, apreciaba el prestigio y el simbolismo otorgados a dichos elementos en sus territorios de origen inmediato y en sus lugares de manufacturación.

Ahora bien, ¿de dónde proceden tanto la cuenta oculada vitrificada como sus cuentas de ámbar acompañantes? Una aproximación a este interrogante se halla en nuestro trabajo sobre las piezas suntuarias de ámbar y pasta vítrea de Cataluña y sus homólogas ibéricas de la Edad del Bronce (Rovira Port, 1995). De hecho, es bien sabido que tanto determinados talleres micénicos continentales como insulares, pudieron proveer de este tipo de objetos suntuarios a un buen número de grupos culturales europeos y, concretamente, peninsulares, ya fuese mediante rutas directas ya fuese a través de factorías o comunidades intermediarias situadas en distintos puntos del Mediterráneo central, caso de las islas de Cerdeña o Sicilia. No obstante, simultáneamente al declive micénico continental, es muy probable que como señalan distintos autores, ya desde el siglo XIII a.C., el mundo chipriota y otros territorios emergentes (Sherratt y Sherratt, 1991; RuizGálvez, 1993) tomasen la iniciativa del comercio y el transporte de distintas mercancías, de entre las cuales, las suntuarias, ocuparían un lugar no menospreciable. Así, los hallazgos arqueológicos demuestran que, tanto a lo largo de todo el segundo milenio como durante las primeras centurias del primero antes de nuestra Era, las vías de comunicación y los canales seculares de comercialización, en absoluto fueron yugulados por coyunturales turbulencias zonales, sino que se mantuvieron inalterables con los altibajos propios de cualquier actividad comercial o transaccional a larga distancia. Y así, la secular asociación de elementos de ámbar y pasta vítrea presente en innumerables yacimientos europeos y peninsulares durante el segundo milenio antes de la Era, se mantiene y resurge con fuerza a lo largo del Bronce Final y en los períodos formativos de nuestras comunidades protohistóricas, como hemos tenido ocasión de comprobar en el yacimiento de La Peña Negra de Crevillente (Alicante) entre los siglos IX/VIII a.C. (González Prats, 1985: 170).

En resumen, los depósitos funerarios presentes en el túmulo I de $\mathrm{El}$ Bosc de Correà nos muestran un ambiente de cultura material que encaja bien con un horizonte avanzado del Bronce Medio zonal. Quizás una datación entre 1600 y 1400 a.C. se aproximaría suficientemente a la etapa final de su utilización y señalaría el lapso cronológico en el cual habría que enmarcar la llegada de los elementos suntuarios reseñados y, específicamente, de la cuenta oculada. En todo caso, aunque es difícil precisar su lugar de origen, no es menos cierto que los puntos de procedencia pudieron ser numerosos: Chipre, Cerdeña, Sicilia, o quizás centros continentales, fueron tal vez zonas primarias de elaboración o pudieron haber disfrutado también del papel de territorios intermediarios.

\section{BIBLIOGRAFÍA}

AA.VV. (e.p.): Autour de l'oeil dans l'Antiquité. Approche pluridisciplinaire. Table Ronde. Lons-le-Saunier, 1994.

Bosch Gimpera, P. (1919): Prehistòria catalana. Enciclopèdia Catalana, XVI. Ed. Catalana. Barcelona: 99126.

- (1915-1920): "L'estat actual del coneixement de la civilització neolítica i eneolítica de la Península Ibèrica". Anuari de l'Institut d'Estudis Catalans, VI: 516-527.

Bosch Gimpera, P. y Pericot, LL. (1915-1920): "Consideracions generals sobre els megàlits catalans". Anuari de l'Institut d'Estudis Catalans, VI: 505-510.

CASTElls, J.; EnRich, J. y ENRICH, J. (1983): “Túmul I de la Serra de Clarena". Excavacions Arqueològiques a Catalunya, 4: 55-81.

CAzurro, M. (1912): Los monumentos megalíticos de la provincia de Gerona. Centro de Estudios Históricos. Madrid.

Charles, R.P. y Guilaine, J. (1963)a: "Découvertes d'objets d'importation orientale dans un site du Bronze Moyen en Languedoc: la grotte au Collier, près de

T. P., 53, n. ${ }^{\circ} 1,1996$ 
Lastours (Aude)". Cahiers Ligures de Préhistoire et d'Archéologie, 12, IIème partie: 205-209.

- (1963)b: "Une grotte sépulcrale du bronze moyen en Languedoc: la Grotte au Collier, commune de Lastours (Aude)". Gallia Préhistoire, VI: 149-163.

CURA, M.; Rovira PORT, J. y Vilardell, R. (1991): “Orígen i filiació dels primers grups tumulars catalans". Actas del Congreso Internacional de Historia de los Pirineos (Cervera, 1988). Madrid: 243-259.

GonzÁlez Prats, A. (1985): "Los nuevos asentamientos del final de la Edad del Bronce: Problemática cultural y cronológica". Arqueología del País Valenciano: panorama y perspectivas. Anejo de Lucentum. Alicante: 153-184.

Gudiol, J. y COLOMINAs, J. (1923): "Sepulcres megalítics de l'Ausetània". Quaderns d'Estudi, XV. Barcelona.

PERICOT, LL. (1925): La civilización megalítica catalana y la cultura Pirenaica. Universidad de Barcelona. Barcelona.

- (1950): Los sepulcros megalíticos catalanes y la cultura pirenaica. Ed. Instituto de Estudios Pirenaicos. Prehistoria y Arqueología, 4. Barcelona.

Rovira PorT, J. (1982): "El fenómeno megalítico en el contexto de la Edad del Bronce del Cispirineo Orien- tal". VII Congreso Internacional de Estudios Pirenaicos (Seu d'Urgell, 1974) Pirineos, 115: 5-24. Jaca.

- (1995): “Ambar y pasta vítrea. Elementos de prestigio entre el Neolítico avanzado y el Bronce Final del nordeste de la Península Ibérica. Un primer estado de la cuestión". Quaderns de Prehistòria i Arqueologia de Castelló, 16: 67-92.

Rovira PORT, J. y CURA MORERA, M. (1989): “El món tumular català des del Bronze Antic fins època ibèrica. Continuitat versus substitució". Actas Congrès Internacional d'Història dels Pirineus (Cervera, 1988). Espacio, Tiempo y Forma, 2: 153-171. Madrid.

Ruiz-GÁlvez, M. (1993): "El occidente de la Península Ibérica, punto de encuentro entre el Mediterráneo y el Atlántico a fines de la Edad del Bronce". Complutum, 4: 41-68.

SERRA VILARO, J. (1927): La civilització megalítica a Catalunya. Contribució al seu estudi. Musaeum Archaeologicum Dioecesanum: 183-188. Solsona.

Sherratt, A. y SherratT, S. (1991): "From luxuries to commodities: the nature of Mediterranean Bronze Age Trading Systems". En N.H. Gale (ed.): Bronze Age trade in the Mediterranean. Studies in Mediterranean Archaeology, XC: 351-386. 